\title{
THE CANADIAN LONG BASELINE ARRAY ${ }^{+}$
}

T.H. Legg,

National Research Council of Canada, Ottawa

On 1983 May 31, the National Research Council of Canada gave formal approval to a new national facility, the 'Canadian Long Baseline Array'. The instrument will be made up of eight radio telescopes spaced across southern Canada, with a ninth antenna, primarily for geophysics, in the north. Though the project is still some way from being funded, the NRC approval was an important and encouraging step in a process that started five years ago.

The Canadian Long Baseline Array (CLBA) began in 1978 with the submission of a proposal to a committee of the Canadian Astronomical Society (CAS). Soon after, the CAS adopted an expanded version of the proposal, with the recommendation that the project be given the highest priority for a new astronomical instrument for Canada. In the years following, the CAS has continued its strong support for the project.

Since 1980, the array has been the work of the 'CLBA Planning Committee' of the CAS. This committee has drawn on the efforts of more than 45 radio and optical astronomers, geophysicists, and engineers in producing a design for the array. The work is summarized in a report published in 1982*, which includes design, reliability and cost studies done under contract by Canadian industry. The description of the CLBA that follows is largely abstracted from this report.

* The report: 'The Canadian Long Baseline Array', dated 1982 September 27 , is to the Natural Sciences and Engineering Research Council and the National Research Council, from the Canadian Astronomical Society.

+ Discussion on page 468

R. Fanti et al. (eds.), VLBI and Compact Radio Sources, 383-389.

() 1984 by the IAU. 


\section{The Overall Telescope}

Two features that are especially emphasized in the design of the CLBA are (i) sensitivity, which is reflected in the choice of the largest antenna affordable, and (ii) image forming over as wide a range of angular scales as possible, a feature discussed further under 'Configuration'.

Estimated sensitivities, with receivers attainable in 1982, are shown in Table I, together with the frequencies covered. The two sets of figures are for a single pair of antennas $(\mathrm{N}=2)$, and for coherent operation of the linear array of telescopes $(\mathrm{N}=8)$, which may be possible at the longer wavelengths or with improved atmospheric measurements.

The beamwidths listed are for the $\sim 5000 \mathrm{~km}$ maximum Canadian baseline. These figures would be reduced to $2 / 3$ of their value with the addition of an antenna in France. Considerable interest has been expressed amongst French scientists, particularly those in the Groupe de Recherches Géodésie Spatiale, in having their planned $32 \mathrm{~m}$ antenna used, as first priority, in cooperation with the CLBA. The French site is essentially ideal from the point of view of the U-V plane coverage.

TABLE I

\begin{tabular}{lccccc}
$\begin{array}{c}\text { Frequency } \\
(\mathrm{GHz})\end{array}$ & \multicolumn{2}{c}{$\mathrm{N}=2$} & \multicolumn{2}{c}{$\mathrm{N}=8$} & \\
& $(\mathrm{mJy})$ & \multicolumn{2}{c}{$\begin{array}{c}\text { BJy }) \\
\text { marcsec }\end{array}$} \\
\hline 0.611 & $\mathrm{sec}$ & $15 \mathrm{~min}$ & $15 \mathrm{~min}$ & 12 hours & \\
1.5 & 90 & 3.0 & 0.57 & 0.082 & 12.2 \\
2.25 & 41 & 1.4 & 0.26 & 0.037 & 5.0 \\
5.0 & 45 & 1.5 & 0.28 & 0.041 & 3.3 \\
8.3 & 51 & 1.7 & 0.32 & 0.046 & 1.5 \\
10.7 & 70 & 2.3 & 0.44 & 0.064 & 0.9 \\
22.0 & 76 & 2.5 & 0.48 & 0.068 & 0.7 \\
\hline
\end{tabular}

2. The Configuration

Eight of the CLBA antennas will form a nearly linear array, running from Newfoundland to British Columbia, but slightly tilted from an exact east-west line. The linear configuration was chosen instead of a two-dimensional array only after much weighing of relative advantages. Scientifically the choice seemed evenly balanced between a wide field-ofview (i.e. sensitivity to a wide range of angular scales) on the one hand, and coverage of low declinations on the other. The more economical maintenance of a line of southern stations turned out to be the deciding factor. 
In arriving at suitable configurations, the image forming properties of different arrays were compared quantitatively by simulating the observation of a number of test sources. Two configurations selected in this way are shown in Fig. 1 , with corresponding $U-V$ curves in Fig. 2 and an example of the imaging tests in Fig. 3. Other test sources used were an extragalactic source with jets, and multiple sources, for simultaneously testing the large and small scale imaging properties of the arrays.

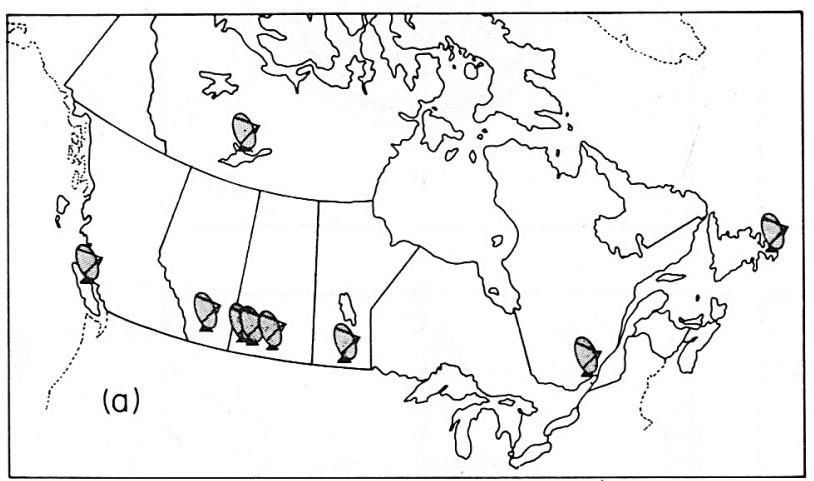

Fig. 1 - Two array configurations designated (a) J3M and (b) Pl0, that fulfill the CLBA design aims. Array Plo uses existing radio telescope sites (but not the telescopes) at Penticton, B.C. and in Algonquin Park, Ontario.

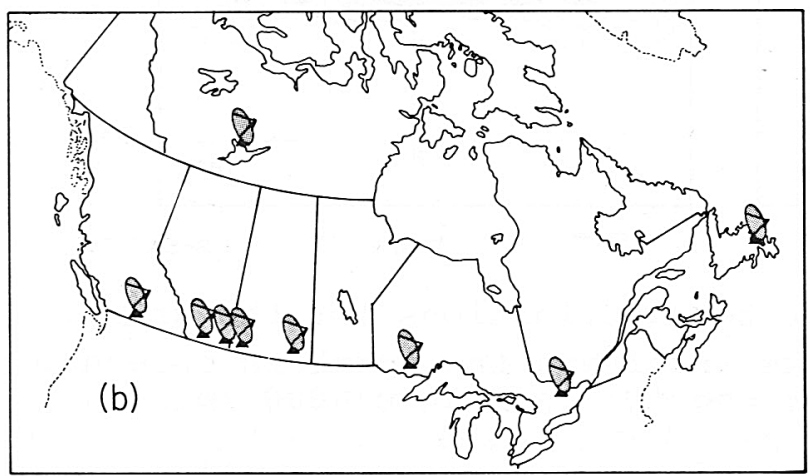

A primary aim of the imaging tests was an array with the widest field-of-view consistent with good imaging. Configurations that best satisfied this aim were found to be those with roughly an exponential distribution of baseline lengths. The arrays of Fig. I are examples. These arrays have minimum baselines of about $75 \mathrm{~km}$, and are able to map sources as large as 1.0 arcsec, at $2.2 \mathrm{GHz}(0.1$ arcsec at $22 \mathrm{GHz}$ ) with the dynamic range (peak brightness to peak brightness error) remaining above 300 .

Other advantages of exponential configurations are: geometrically similar sampling of the visibilities of similar sources of different angular scale, and a near uniform 'filling-in' of the $U-V$ plane for a moderate (say $\sim 10 \%$ ) 
bandwidth. The tilt of the arrays of Fig. I from an eastwest line also slightly improves the filling-in of the U-V plane. After the first 12 hours of observations, the $\mathrm{U}-\mathrm{V}$ tracks interleave instead of overlapping.
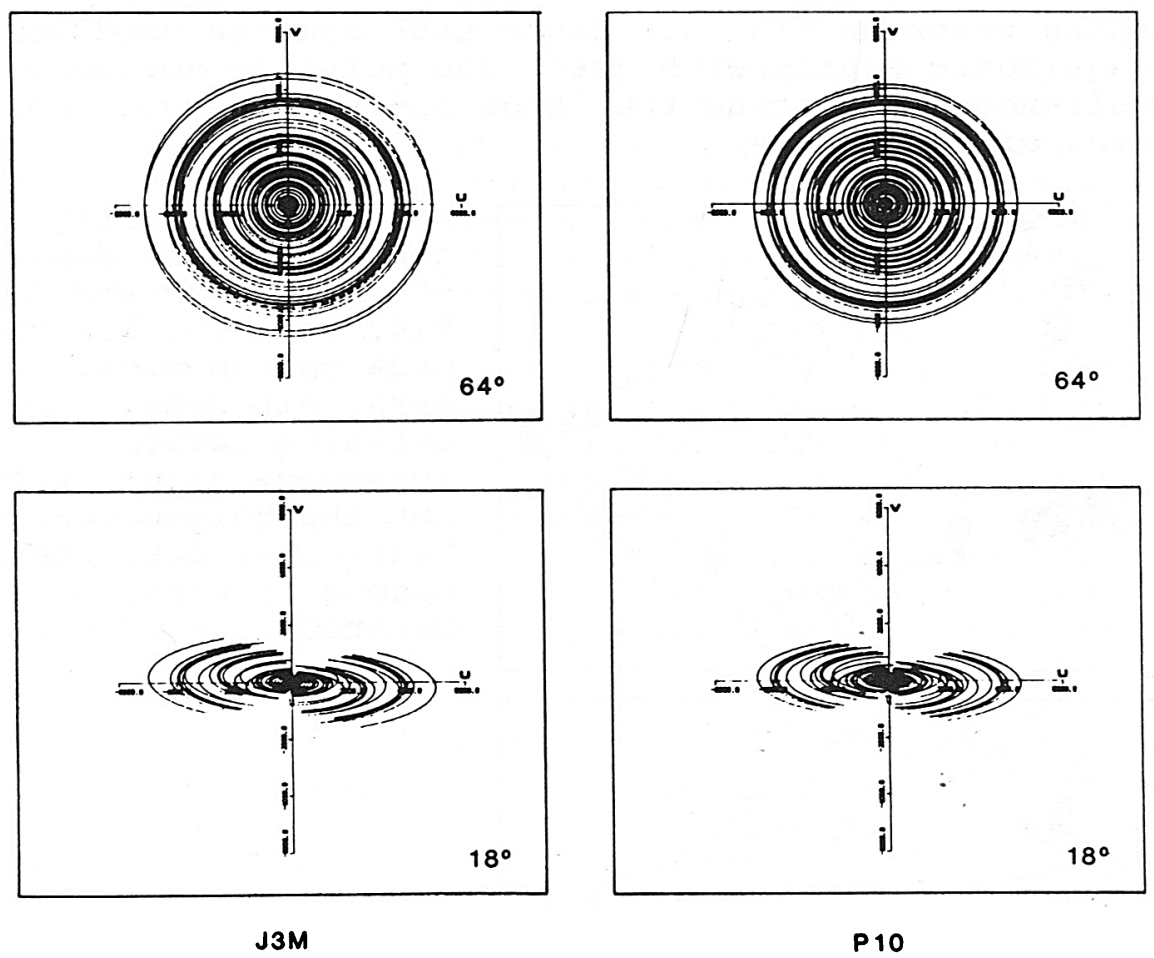

Fig. 2 - The U-V tracks at two declinations for the arrays of Fig. 1, excluding tracks involving the northern antenna. The dimensional tick under the 'U' indicates $6000 \mathrm{~km}$.

The eight southern antennas of the linear array will be used primarily for astronomy, while the ninth antenna, at Yellowknife, Northwest Territories, will provide northsouth baselines which are a priority of Canadian geophysicists. A second geophysical priority will be satisfied by having baselines on, and close to, the geologically stable Canadian shield.

\section{Antennas}

The $32 \mathrm{~m}$ dia antennas of the CLBA will be upgraded versions of a standard wheel-and-track communications antenna. The antenna will have 'shaped' main and secondary reflectors to give near uniform illumination (i.e. little apodization) and, consequently, high efficiency ( $\vec{\approx} 70 \%)$. At the time of the CLBA report, the highest frequency considered was 
$22 \mathrm{GHz}$. Since then, the possibility of improved panels, to give a useful collection area at $43 \mathrm{GHz}$, has been considered. Any such improvements would have to be made, however, to fit within the overall cost estimates of the 1982 CLBA report.
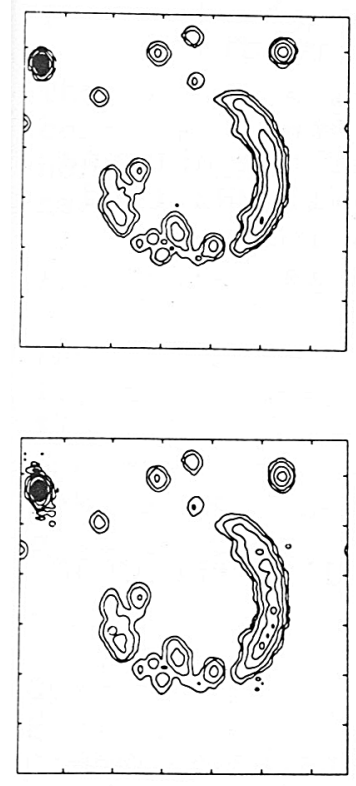

$\operatorname{san}$
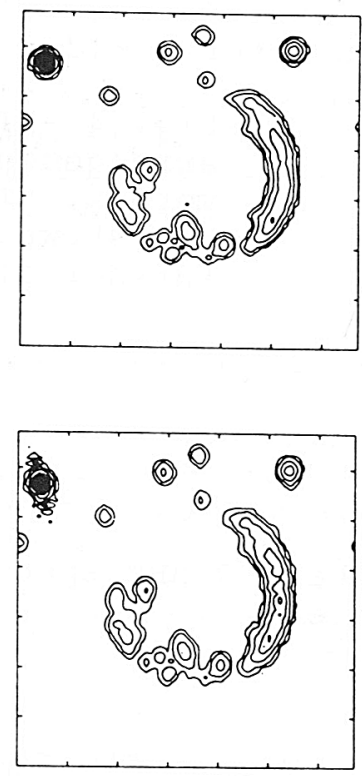

DIO

Fig. 3 - Reproduced images from simulated observations with arrays J3M and P10, for declinations $64^{\circ}$ (top) and 180 (bottom). The original, a DRAO map of CTBI, is on the right. The lowest contour level is 0.002 of the peak brightness.

\section{Antenna Feeds}

Dual polarized feeds for each of the frequencies listed in Table I will be arranged at the vertex of the $32 \mathrm{~m}$ antenna, as shown in Fig. 4. The feed will be selected by appropriately tilting the antenna subreflector. A dual frequency feed will be used for the 2.25 and $8.3 \mathrm{GHz}$ bands. The 611 MHz feed, not shown in Fig. 5, will be a large uncorrugated horn of square aperture, placed to one side of the vertex. The length of this horn is kept manageable by having a flare angle that varies with distance from the throat. 


\section{Receivers}

Dual GaAs FET amplifiers will be used at all of the frequencies listed in Table I, and cooled (except at 611 $\mathrm{MHz}$ ) to $20 \mathrm{~K}$. The development of such amplifiers is underway at the University of Alberta and the National Research Council, Ottawa. The local oscillators will be phaselocked to a station hydrogen maser. Wider intermediate

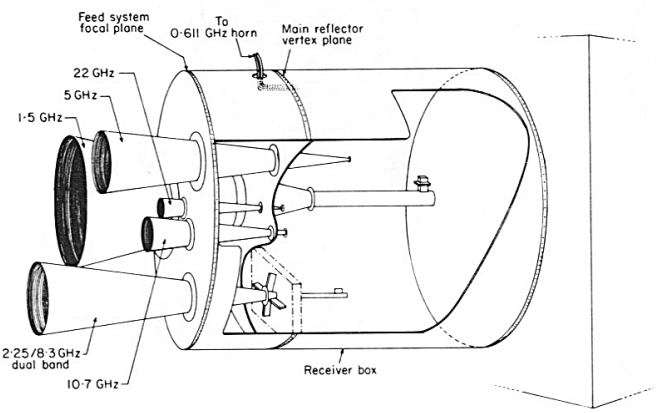

Fig. 4 - The feed arrangement at the antenna vertex. The $611 \mathrm{MHz}$ feed, not shown, is located further off axis.

frequency bandwidths than the $200 \mathrm{MHz}$ specified in the CLBA report are now being discussed.

\section{Recorders}

Video recorders with automatic cassette changers have been specified since the 1978 proposal. The 1982 design calls for two channels, of 4 recorders each, recording a total of $96 \mathrm{M}$ bits sec-1. These would run unattended for 24 hours. This bandwidth will possibly be increased as a result of rapid advances in the recorder field. Reliability of the cassette machines, which was already good, has been improved by the development of automatic equalizers by J.L. Yen at the University of Toronto.

\section{Correlators}

The correlators will receive data streams from up to 10 antennas. Normally, 32 lags will be produced for each of four polarization products. Fractional-bit delay correction and three-level 'fringe rotation' schemes will be incorporated. Up to 2048 spectral line channels will be provided by recirculating the data to the correlators.

\section{Image Processing}

Image processing for the CLBA is designed to give the nonexpert user a source image, a spectrum, or geophysical data without necessarily any direct intervention on his part. The processing is divided into levels in which, typically: 
(i) edited and calibrated correlation coefficients are produced; (ii) images are produced, and (iii) images are enhanced in various ways. The design centers upon a VAX $11 / 780$ computer and three PDP 11/24 computers controlling array processors.

\section{Operation}

The antennas will be controlled over telephone lines from a central computer and will be unattended except for a few hours a day. Teams from the headquarters will undertake nearly all of the maintenance.

\section{Staff and Costs}

The staff of the CLBA will total 75, as indicated in Table II; and construction costs will total an estimated $\$ 70.1 \times 10^{6}$, distributed as shown in Table III. Yearly operating costs will be $\$ 7.7 \times 10^{6}$ of which salaries will make up $\$ 3 \times 10^{6}$ and the purchase of new receivers, or other equipment to upgrade the instrument, $\$ 1.5 \times 10^{6}$.

TABLE II

\section{CLBA STAFF}

Operations

Engineering support

Computer programming

Administration and services

Development

Scientists

Antennas and feeds

(Millions of 1982 Canadian dollars)

Receivers and electronics

10.2

Correlation and processing

6.4

Headquarters

5.2

Sites

4.4

Design, management, other

5.8

Contingency

TOTAL

$\frac{6.4}{70.1}$ 